\title{
Weibull Failure Probability Estimation Based on Zero-Failure Data
}

\author{
Ping Jiang, ${ }^{1}$ Yunyan Xing, ${ }^{2}$ Xiang Jia, ${ }^{1}$ and Bo Guo ${ }^{1}$ \\ ${ }^{1}$ College of Information System and Management, National University of Defense Technology, Changsha 410073, China \\ ${ }^{2}$ College of Continuing Education, National University of Defense Technology, Changsha 410073, China
}

Correspondence should be addressed to Ping Jiang; jiangping@nudt.edu.cn

Received 27 May 2014; Revised 4 September 2014; Accepted 15 October 2014

Academic Editor: Wenbin Wang

Copyright (c) 2015 Ping Jiang et al. This is an open access article distributed under the Creative Commons Attribution License, which permits unrestricted use, distribution, and reproduction in any medium, provided the original work is properly cited.

Reliability testing is often carried out with small sample sizes and short duration because of increasing costs and the restriction of development time. Therefore, for highly reliable products, zero-failure data are often collected in such tests, which could not be used to evaluate reliability by traditional methods. To cope with this problem, the match distribution curve method was proposed by some researchers. The key step needed to exercise this method is to estimate the failure probability, which has yet to be solved in the case of the Weibull distribution. This paper presents a method to estimate the intervals of failure probability for the Weibull distribution by using the concavity or convexity and property of the distribution function. Furthermore, to use the method in practice, this paper proposes using the approximate value of the shape parameter determined by either engineering experience or by hypothesis testing through a $p$ value. The estimation of the failure probability is thus calculated using a Bayesian approach. A numerical example is presented to validate the effectiveness and robustness of the method.

\section{Introduction}

Reliability testing is usually required in product development to evaluate product reliability. Product lifetimes are becoming longer than in previous decades because of the improvement of reliability. Therefore, with the restrictions of increasing costs and short development times, reliability testing is often carried out with small sample sizes and short duration, which will often lead to zero-failure data [1]. Accordingly, it is desirable to estimate product reliability using the zero-failure data.

The zero-failure scenario is described as follows.

Let $F(t, \theta)$ denote the lifetime distribution of a product, where $\theta \in \Theta$ is the parameter of the distribution and $\Theta$ is the parameter space. The reliability test is composed of $k$ truncated tests with corresponding censored time $t_{i}(i=1,2, \ldots, k)$, which satisfies $t_{1}<t_{2}<\cdots<t_{k}$. The sample size for the $i$ th test is $n_{i}$. As no failure is observed in the tests, the zero-failure data is expressed by $\left(t_{i}, n_{i}\right), i=$ $1,2, \ldots, k$. Let $p_{i}=P\left(T \leq t_{i}\right)=F\left(t_{i}\right)$ denote the failure probability of the product at time $t_{i}$. Then $p_{i}$ satisfies the following statements:

(1) $p_{0}=0$, when $t=0$;

(2) $p_{1}<\cdots<p_{k}$

Let $s_{i}=\sum_{j=i}^{k} n_{j}$ denote the number of samples at time $t_{i}$; that is, there are $s_{i}$ samples in the reliability test at time $t_{i}$.

Estimating product reliability based on zero-failure data is challenging. Welker and Lipow [1] first raised this problem and, since then, some researchers have made progress on the topic [2,3]. For the binomial distribution, Bailey [4] proposes a model to predict failure probability from zerofailure data; however, his model requires a large sample size. Based on zero-failure data, Wang and Langanke [5] present an approach that compares the reliability index-mean time to failure (MTTF)-between a newly designed product and the old product, but their approach is based on the assumption that the shape parameters for the two products are the same, which limits the application in practice. Miller et al. [6] 
study the estimation of failure probability for software when no errors are observed in testing. Jiang et al. [7] construct the shrinkage preliminary test estimator (SPTE) to estimate the reliability of a product following a Weibull distribution, when a prior estimate is available. Chen et al. [8] introduce the optimal confidence limit method to obtain the optimal lower confidence limit of some parameters of reliability distributions, in the case of zero-failure data. However, their method only considers one sample in each test and could not be applied to the case of multiple samples in each test. As no failure is observed in the tests, the maximum likelihood estimation (MLE) approach could not be applied in such a scenario. To solve this problem, L. Wang and B. Wang [9] propose the modified maximum likelihood estimation (MMLE), introducing a new parameter, $L$, to modify the results obtained by applying MLE on the zero-failure data. The key to MMLE is the value of parameter L. However, some researchers have found that the parameter, $L$, will often cause the overestimation of parameters. The overestimation usually results from the improper use of the zero-failure data, which has raised debates among researchers for a long time. One solution is to introduce failure information when dealing with zero-failure data [10]. The key to this method is to acquire the failure information (i.e., failure time), whereas it is often estimated by the zero-failure data. This will result in an inaccurate estimate when the test duration is far less than the real lifetime of a product [11]. Mao and Luo [12] present the match distribution curve (MDC) method to solve the evaluation on zero-failure data: first, estimating the failure probability $p_{i}$ at censoring time $t_{i}$ is carried out; then, the data pairs $\left(t_{i}, p_{i}\right)$ are used to construct a distribution curve to estimate the parameters of the distribution; finally, the reliability can be evaluated based on the distribution. The MDC method is widely used in various distributions with zero-failure data; however, it is not discussed in the case of a Weibull distribution because of the computational complexity of the distribution. Motivated by this problem, we focus our research on the failure probability estimation method in a Weibull distribution.

\section{Weibull Distribution}

When evaluating reliability using test data, we often assume that the product life follows some kind of distribution. Then, the parameters of the distribution are estimated based on the data, and reliability is evaluated by using the determined distribution. The Weibull distribution is one of the most commonly used distributions in reliability evaluation because of its ability to take on various forms by adjusting its parameters [13]. The two-parameter Weibull distribution is defined as

$$
f(t)=\frac{m}{\eta}\left(\frac{t}{\eta}\right)^{m-1} \exp \left[-\left(\frac{t}{\eta}\right)^{m}\right] \quad(t>0),
$$

where $m$ is the shape parameter and $\eta$ is the scale parameter. The CDF of the Weibull distribution is defined as

$$
F(t)=P(T \leq t)=1-\exp \left[-\left(\frac{t}{\eta}\right)^{m}\right] .
$$

The failure rate function $\lambda(t)=m / \eta(t / \eta)^{m-1}$ is an increasing function, when $m>1$, which describes the character of various products. The Weibull distribution will become another type of distribution when the value of shape parameter $m$ varies. For example, it becomes an exponential distribution when $m=1$; it becomes a Rayleigh distribution when $m=2$; and it approximates a normal distribution when $m \in[3,4]$. Ning [10] summarizes that a Weibull distribution can be used to describe traumatic failures, when $m \leq 1$, and it can be applied to describe degradation failures, when $m \geq 3.25$. Therefore, it can be adopted to describe the combined effects of traumatic failures and degradation failures, when $m \in(1,3.25)$, where the ratio of the two failures is determined by the value of $m$. Due to the flexibility of Weibull distribution, it is widely used in reliability evaluation in practice, even in the cases of zero-failure data.

As the key step in the MDC method is failure probability estimation, which is yet to be solved in the case of a Weibull distribution, we present this estimation procedure in the following section.

\section{Failure Probability Estimation}

Let $y=\ln \ln (1 / 1-F), x=\ln t$, and $b=m \ln \eta$; then (2) is transformed into

$$
y=m x-b
$$

The linear regression method could be applied to calculate the parameters of the Weibull distribution. Here, the weighted least square estimation method is used to obtain the estimates of parameters $\widehat{m}$ and $\hat{\eta}$, which minimizes

$$
Q=\sum_{i=1}^{k} w_{i}\left(y_{i}-m x_{i}+m \ln \eta\right)^{2}
$$

As it is already proven that the weighted least square estimates, $\widehat{m}$ and $\widehat{\eta}$, are unbiased, we propose the use of the method and briefly introduce it here.

Denote the weight $w_{i}=n_{i} t_{i} / \sum_{i=1}^{k} n_{i} t_{i}(i=1,2, \ldots, k)$, where $t_{i}$ is failure time. Let $A=\sum_{i=1}^{k} w_{i} x_{i}, B=\sum_{i=1}^{k} w_{i} x_{i}^{2}$, $C=\sum_{i=1}^{k} w_{i} y_{i}$, and $D=\sum_{i=1}^{k} w_{i} x_{i} y_{i}$. The weighted least square estimates, $\widehat{m}$ and $\hat{\eta}$, are obtained by referring to

$$
\begin{gathered}
\widehat{m}=\frac{B-A^{2}}{D-A C}, \\
\widehat{\eta}=\exp \left(\frac{B C-A D}{B-A^{2}}\right) .
\end{gathered}
$$

After $\widehat{m}$ and $\widehat{\eta}$ are acquired, given the test time $t_{i}$, it is easy to calculate the failure probability at time $t_{i}$ for the Weibull distribution by

$$
p_{i}=F\left(t_{i}\right)=1-\exp \left[-\left(\frac{t_{i}}{\widehat{\eta}}\right)^{\widehat{m}}\right] .
$$

The above procedure is typical to obtain the failure probability at time $t_{i}$ for the Weibull distribution given 
that $t_{i}(i=1,2, \ldots, k)$ is the corresponding failure time. To calculate the failure probability at time $t_{i}$ for the Weibull distribution with zero-failure data, we have to consider an alternative solution. This is discussed in the next subsection for Weibull distribution.

3.1. Failure Probability Estimation with Zero-Failure Data. In cases of zero-failure data, Ning [10] proposes the following equation to estimate $p_{i}$ at censoring time $s_{i}$ :

$$
\widehat{p}_{i}=\frac{0.5}{s_{i}+1} \quad(i=1,2, \ldots, k) .
$$

Equation (7) is designed to calculate the mean value of the upper limit $1 /\left(s_{i}+1\right)$ and lower limit 0 , which is too simple and arbitrary, and often results in inaccurate estimation.

Bayesian theory is the most popular method to estimate $p_{i}$ as it combines prior information with test data, making the estimation more accurate than if only prior information or test data was used alone. In Bayesian theory, distribution parameter $\theta$ is regarded as a random variable, and the prior distribution is determined by using historical data, experts' judgments, or data from similar products to obtain the posterior distribution of $\theta$ by

$$
\pi(\theta \mid x)=\frac{\pi(\theta) f(x \mid \theta)}{\int_{\theta} \pi(\theta) f(x \mid \theta) d \theta},
$$

where $f(x \mid \theta)$ is the PDF of the population $X$, or it can be replaced by likelihood function $L(\theta)$, and $\pi(\theta)$ is the prior distribution of $\theta$. The point estimate of $\theta$ is $\widehat{\theta}=$ $\int_{\theta} \theta \pi(\theta \mid x) d \theta$, which is the expectation of the posterior distribution under the square loss.

In the case of zero-failure data, to estimate $p_{i}$, the likelihood function is usually defined as $L=\left(1-p_{i}\right)^{s_{i}}$. Therefore, in terms of Bayes' theorem, the key to estimating $p_{i}$ is to choose the prior distribution of $p_{i}$. To achieve this, two important answers should be sought here: the right choice of the prior distribution and the determination of the interval of $p_{i}$ in the prior distribution.

(1) The Right Choice of the Prior Distribution of $p_{i}$. For the choice of the prior distribution of $p_{i}$, one may simply assume that $p_{i}$ follows the uniform distribution. However, since the data exhibits zero failure, the value of failure probability $p_{i}$ is more likely to be small. Therefore, Han and $\mathrm{Li}$ [14] present the idea of utilizing the decreasing function to construct the prior distribution. We also adopt this idea and use it to construct the prior distribution in the next section.

(2) The Interval of $p_{i}$. To determine the interval of $p_{i}$ for a normal distribution, Zhang [15] proves that the CDF of $F(t)$ is a concave function in $t$, when $t<\mu$. Then, based on the properties of the concave function, Zhang concludes that the following inequalities stand:

$$
0<\frac{p_{1}}{t_{1}}<\frac{p_{2}}{t_{2}}<\cdots<\frac{p_{k}}{t_{k}}<\frac{p_{u}}{t_{k}} .
$$

Then the interval of $p_{i}$ is $\left[0,\left(t_{i} / t_{k}\right) p_{u}\right]$, where $p_{u}$ is the upper limit preset by expert or engineering experience.
Inspired by this theory, and given the distribution type, researchers tried to use the properties of the distribution to determine the interval of $p_{i}$ if there is no other prior information: in the case of an exponential distribution, Ning [10] obtains the interval of $p_{i}$ as $\left[p_{i-1},\left(t_{i} / t_{i-1}\right) p_{i-1}\right]$, and in the case of an extreme distribution, $\mathrm{Li}$ [11] obtains the interval of $p_{i}$ as $\left[p_{i-1},\left(t_{i} / t_{k}\right) p_{u}\right]$. However, in the case of a Weibull distribution, there is no reference to determine the interval of $p_{i}$, which is why we choose to study this problem according to the convex and concave properties of the Weibull distribution.

3.2. Weibull Failure Probability Estimation. Based on the accumulated test data from Weibull-distributed products in worldwide practice, Han [16] states that the shape parameter, $m$, for Weibull-distributed products is usually within the interval of $[1,10]$. So, the interval for $m$ is set to be $[1,10]$.

To obtain the convex and concave properties of the Weibull distribution, by taking the second derivative of (2), we have

$$
\frac{d^{2} F}{d t^{2}}=\frac{m t^{m-2} \exp \left(-(t / \eta)^{m}\right)}{\eta^{m}}\left(m-1-\frac{m t^{m}}{\eta^{m}}\right) .
$$

From (10), it is easy to find that $d^{2} F / d t^{2}<0$, when $0<m \leq 1$, and when $m>1$, let the inflexion point $t_{\text {turn }}=$ $\eta((m-1) / m)^{1 / m}$; then $d^{2} F / d t^{2}>0$, when $t \in\left[0, t_{\text {turn }}\right)$; $d^{2} F / d t^{2}<0$, when $t \in\left(t_{\text {turn }},+\infty\right)$. So, according to the convex and concave criteria, we have the following: $F(t)$ is convex, when $0<m \leq 1$, and $F(t)$ is concave, when $m>1$ and $t \in\left[0, t_{\text {turn }}\right)$. This conclusion provides us with the criteria for determining the interval of $p_{i}$.

(1) When $0<m \leq 1, F(t)$ is convex, so we have

$$
\frac{p_{1}}{t_{1}}>\frac{p_{2}}{t_{2}}>\cdots>\frac{p_{i-1}}{t_{i-1}}>\frac{p_{i}}{t_{i}}>\cdots>\frac{p_{k}}{t_{k}}
$$

As we have assumed that $p_{i-1}<p_{i}$, the following inequality stands:

$$
p_{i-1}<p_{i}<\frac{t_{i}}{t_{i-1}} p_{i-1} \quad(i \geq 2) .
$$

Therefore, the interval of $p_{i}$ is $\left(p_{i-1},\left(t_{i} / t_{i-1}\right) p_{i-1}\right)$, which could be calculated by an iterative method beginning from $p_{1}$ $(i=1)$.

The calculation begins with $p_{1}$, which can be estimated by the Bayesian method. Let the interval of $p_{1}$ be $\left(0, p_{\text {upper }}\right)$, where $p_{\text {upper }}$ is the upper limit set by experts. In practice, $p_{\text {upper }}=0.5$ is often used. However, this value of $p_{\text {upper }}$ is conservative here, as no failure occurs; we can assume that $p_{1}<p_{i} \leq 0.5(i \geq 2)$, so $p_{\text {upper }}$ could be set at less than 0.5 .

(2) When $m>1, F(t)$ is not strictly convex or concave, which makes it difficult to analyze. Therefore, we present our solution as follows.

We can acquire the median time $t_{\text {mid }}=\eta \exp (\ln \ln 2 / \mathrm{m})$ by referring to (1), when $F\left(t_{\text {mid }}\right)=0.5$. The inflexion point (time) is obtained in a similar manner, which is denoted by $t_{\text {turn }}=\eta((m-1) / m)^{1 / m}$. 
As the data reveals no failure, we assume that $t_{1}<\cdots<$ $t_{k}<t_{\text {mid }}$. Therefore, to find the interval, within which $F(t)$ exhibits convexity or concavity, we will determine the interval that satisfies $t_{\text {mid }} \leq t_{\text {turn }}$.

Let $g(x)=\exp (\ln \ln 2 / x)$ and $h(x)=((x-1) / x)^{1 / x}$, where $x \in(1,10]$. Then it is easy to prove that both $g(x)$ and $h(x)$ are strictly increasing in $(1,10]$ and $g(x)=h(x)$, when $x \approx 3.3$, as shown in Figure 1. Therefore, when $m \geq 3.3$, we have $t_{\text {mid }} \leq t_{\text {turn }}$, and $F(t)$ is concave.

When $3.3 \leq m \leq 10, F(t)$ is concave. According to the property of the concave function (as shown in (9)), we have $\left(t_{i} / t_{i-1}\right) p_{i-1}<p_{i}$. Thus, the upper limit of $p_{i}$ needs to be determined. Li [11] and Zhang [15] suggest setting a universal value of the upper limit, $p_{\text {upper }}$, for all $p_{i}$, which, in fact, extends the interval of $p_{i}$ and makes analysis inaccurate. In this paper, we introduce the parameter $p_{\text {con }}$, which satisfies $p_{i}<p_{i-1}+p_{\text {con }}$, where $p_{\text {con }}$ is the possible difference of $p_{i}$ and $p_{i-1}$, that is, $p_{\text {con }} \triangleq \Delta p=p_{i}-p_{i-1}$. As there are $k$ censored tests with zero-failure data, we can assume that the value of $p_{\text {con }}$ is around $0.5 / k$. So, its value is limited to a much smaller range and can be preset by expert or engineering experience. So we have when $m \in[3.3,10]$, $p_{i} \in\left(\left(t_{i} / t_{i-1}\right) p_{i-1}, p_{i-1}+p_{\text {con }}\right)$.

(3) When $1 \leq m \leq 3.3$, there is no similar method to find the concave or convex properties of $F(t)$. We can transform (2) into the following equations, given $p_{i-1}$ and $p_{i}$, respectively:

$$
\begin{gathered}
\ln \ln \frac{1}{1-p_{i-1}}=m \ln t_{i-1}-m \ln \eta \\
\ln \ln \frac{1}{1-p_{i}}=m \ln t_{i}-m \ln \eta .
\end{gathered}
$$

We can combine these two equations to obtain

$$
\ln \frac{\ln \left(1-p_{i-1}\right)}{\ln \left(1-p_{i}\right)}=m \ln \frac{t_{i-1}}{t_{i}}<\ln \frac{t_{i-1}}{t_{i}},
$$

which is equivalent to

$$
\frac{\ln \left(1-p_{i-1}\right)}{t_{i-1}}>\frac{\ln \left(1-p_{i}\right)}{t_{i}} .
$$

Then we can obtain the lower limit of $p_{i}$ as 1 $\left(1-p_{i-1}\right)^{t_{i} / t_{i-1}}$. For the upper limit of $p_{i}$, in a similar manner, let it be $p_{i-1}+p_{\text {con }}$. So here we have, when $1<m<3.3$, $1-\left(1-p_{i-1}\right)^{t_{i} / t_{i-1}}<p_{i}<p_{i-1}+p_{\text {con }}$.

Now, we can determine the intervals of $p_{i}$, the failure probability in prior distribution, with respect to the values of $m$. Denote the interval by $\left(p_{l}, p_{u}\right)$. Let $\left(1-p_{i}\right)^{2}$ denote the core of the prior distribution. To meet the requirement of the distribution (i.e., $\int_{p} A\left(1-p_{i}\right)^{2} d p=1$ ) the PDF of the prior distribution of $p_{i}$ is defined by $\pi\left(p_{i}\right)=$ $3\left(1-p_{i}\right)^{2} /\left(\left(1-p_{l}\right)^{3}-\left(1-p_{u}\right)^{3}\right)$. Then, by referring to (8), we can obtain the PDF of the posterior distribution for $p_{i}$ as $\pi\left(p_{i} \mid s\right)=\left(s_{i}+3\right)\left(1-p_{i}\right)^{s_{i}+2} /\left(\left(1-p_{l}\right)^{s_{i}+3}-\left(1-p_{u}\right)^{s_{i}+3}\right)$.

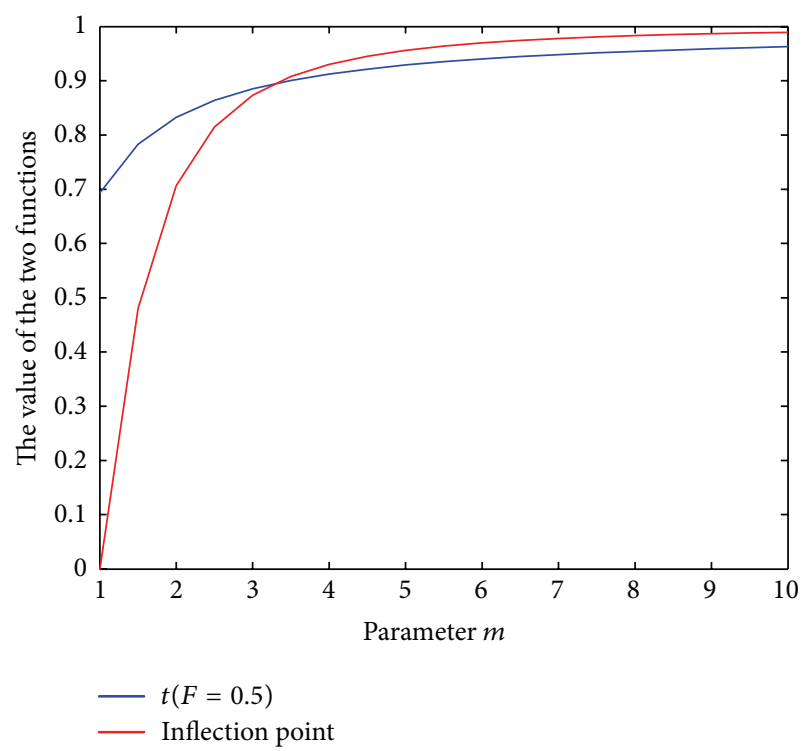

Figure 1: Comparison between abscissas of inflection point and time when failure probability is 0.5 .

Under the square loss assumption, we can acquire the expectation of $p_{i}$ as the estimate as follows:

$$
\widehat{p}_{i}=\frac{s_{i}+3}{\left(1-p_{l}\right)^{s_{i}+3}-\left(1-p_{u}\right)^{s_{i}+3}}\left[q\left(p_{u}\right)-q\left(p_{l}\right)\right]
$$

where

$$
q(x)=\frac{(1-x)^{s_{i}+4}}{s_{i}+4}-\frac{(1-x)^{s_{i}+3}}{s_{i}+3} .
$$

According to this, the interval of $p_{i}$ depends on the value of $m$. But it is not necessary to find the exact value of $m$; only the interval of $m$ is to be determined to calculate the estimate of failure probability.

In our early paper [17], we presented the above estimate without discussing the estimate of $m$. There is a large amount of engineering experience in practice to help us estimate the interval of $m$ [18]. If there is little knowledge that we can refer to, we propose using the following method [19] to determine $m$, which is based on hypothesis testing.

(1) Propose the hypothesis: $H_{0}: m=m_{0} \quad H_{1}: m \neq m_{0}$.

(2) Construct the statistic

$$
G_{k}=\frac{\sum_{i=1}^{k-1} i W_{i+1}}{(k-1) \sum_{i=1}^{k} W_{i}},
$$

where $W_{i}=(k-i+1)\left(t_{i}^{m}-t_{i-1}^{m}\right)(i=1, \ldots, k)$ and $t_{0}=0$. 
TABLE 1: Sample data.

\begin{tabular}{|c|c|c|c|c|c|c|}
\hline Subgroup number & 1 & 2 & 3 & 4 & 5 & 6 \\
\hline Parameters & \multicolumn{6}{|c|}{$m=0.8, \eta=2800$} \\
\hline Censoring time $(\mathrm{h})$ & 33.329 & 34.388 & 141.569 & 417.819 & 657.716 & 778.398 \\
\hline$s$ & 12 & 11 & 10 & 8 & 7 & 6 \\
\hline Parameters & \multicolumn{6}{|c|}{$m=1.8, \eta=2800$} \\
\hline Censoring time $(\mathrm{h})$ & 531.821 & 634.651 & 826.586 & 1029.37 & 1360.66 & 1768.31 \\
\hline$s$ & 12 & 11 & 9 & 7 & 5 & 3 \\
\hline Parameters & \multicolumn{6}{|c|}{$m=3.8, \eta=2800$} \\
\hline Censoring time $(\mathrm{h})$ & 2.38799 & 785.303 & 1428.62 & 1706.08 & 1724.18 & 2237.69 \\
\hline$s$ & 12 & 10 & 9 & 8 & 7 & 5 \\
\hline
\end{tabular}

(3) When $3 \leq k \leq 20$, the rejection interval for $H_{0}$ is $\left\{G_{k}>\zeta_{1-\alpha / 2}, G_{k}<\zeta_{\alpha / 2}\right\}$, where $\zeta_{\alpha / 2}$ is the $100(\alpha / 2) \%$ quantile, and the CDF of $G_{k}$ is defined by

$$
\begin{aligned}
P\left(G_{k} \leq x\right)= & x^{k-1}\left\{\prod_{i=1}^{k-1} c_{i}\right\}^{-1} \\
& -\sum_{j=l+1}^{k-1}\left(x-c_{j}\right)^{k-1}\left\{c_{j} \prod_{r \neq j}^{k-1}\left(c_{r}-c_{j}\right)\right\}^{-1},
\end{aligned}
$$

where $c_{j}=(k-j) /(k-1)$ and $l=\max \left(\arg t \leq c_{l}\right)$.

(4) Let $p$ value $=P\left\{G_{k}>g_{r} \mid m=m_{0}\right\}$, which is maximized, if $m=m_{0}, g_{r}$ is the sample result of $G_{k}$. When $p$ value is maximized, the estimate of $m$ is the best.

From the above introduction to our proposed method, one may find that to estimate the failure probability of a Weibull distribution with zero-failure data, it requires only roughly determining the interval of parameter $m$ (instead of obtaining its exact value). Besides, there is no need to match the exact value of $m$ by the above hypothesis testing method as it greatly increases the complexity of the calculation. Therefore, in practice, if the $p$ value in the hypothesis testing is comparatively large, we can conclude that parameter $m$ is determined.

3.3. Summary of the Method. The proposed method is summarized as follows.

(1) Roughly determine the interval of parameter $m$ in the Weibull distribution. If engineering experience is not available, roughly estimate $m$ by referring to the $p$ value in the hypothesis testing.
(2) Based on the estimate of $m$, determine the interval of failure probability by

$p_{i}$

$$
\in \begin{cases}\left(p_{i-1}, \frac{t_{i}}{t_{i-1}} p_{i-1}\right), & 0<m \leq 1 \\ \left(1-\left(1-p_{i-1}\right)^{t_{i} / t_{i-1}},\right. & \\ \left.p_{i-1}+p_{\text {con }}\right), & 1<m<3.3, p_{1} \in\left(0, p_{\text {upper }}\right) \\ \left(\frac{t_{i}}{t_{i-1}} p_{i-1}, p_{i-1}+p_{\text {con }}\right), & 3.3 \leq m \leq 10\end{cases}
$$

(3) Estimate $p_{i}$ by (16).

\section{A Numerical Example}

To illustrate the validity of our proposed method, it is applied to estimate the failure probability and compared with the true value from a given Weibull distribution. Simulation data is used in this numerical example. There are twelve samples in the example and they will experience six censored tests. We use MATLAB software to generate several groups of random values for twelve variables following a given Weibull distribution and, for each group, randomly divide them into six subgroups. The largest variable in each subgroup is regarded as the censoring time for the subgroup. To ensure that the random variables generated correspond to no failure, the failure probability of each variable should be less than 0.5 . This means that only those groups whose failure probability of the largest variable in each subgroup is less than 0.5 are chosen as our samples. The samples are listed in Table 1.

After the data is obtained, the failure probability at censoring time $t_{i}$ is estimated and is then compared with the true value, calculated by the given Weibull distribution at censoring time $t_{i}$. The results are also compared with those calculated by the classical method in (7). In this example, we assume that the interval of parameter $m$ is predetermined by engineering experience or by our proposed hypothesis testing, and we also try to figure out the effects that $p_{\text {upper }}$ and $p_{\text {con }}$ have on the final estimation.

Figure 2 shows the samples generated by the Weibull distribution with the parameters $m=0.8, \eta=2800$. The 


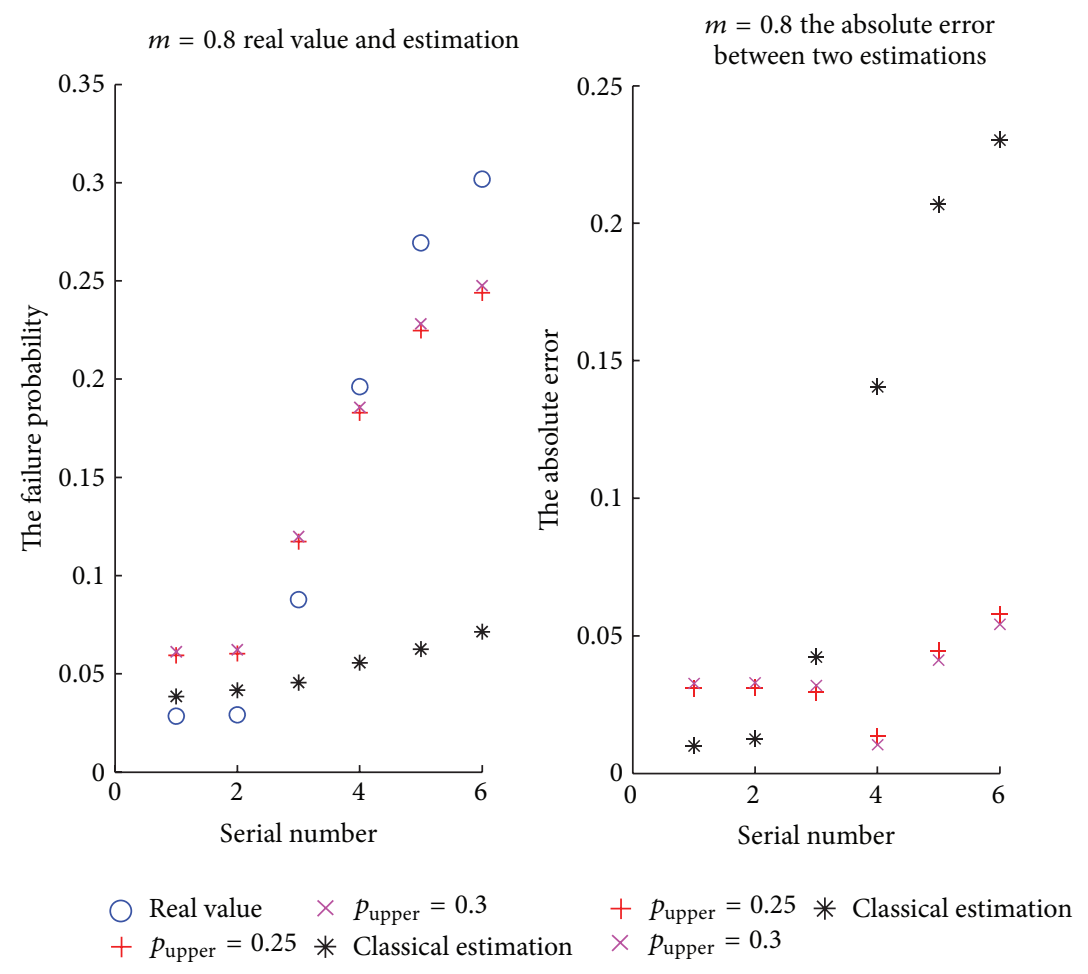

FIGURE 2: Comparison of failure probability among real value, estimator, and classical estimation value when $m=0.8$.

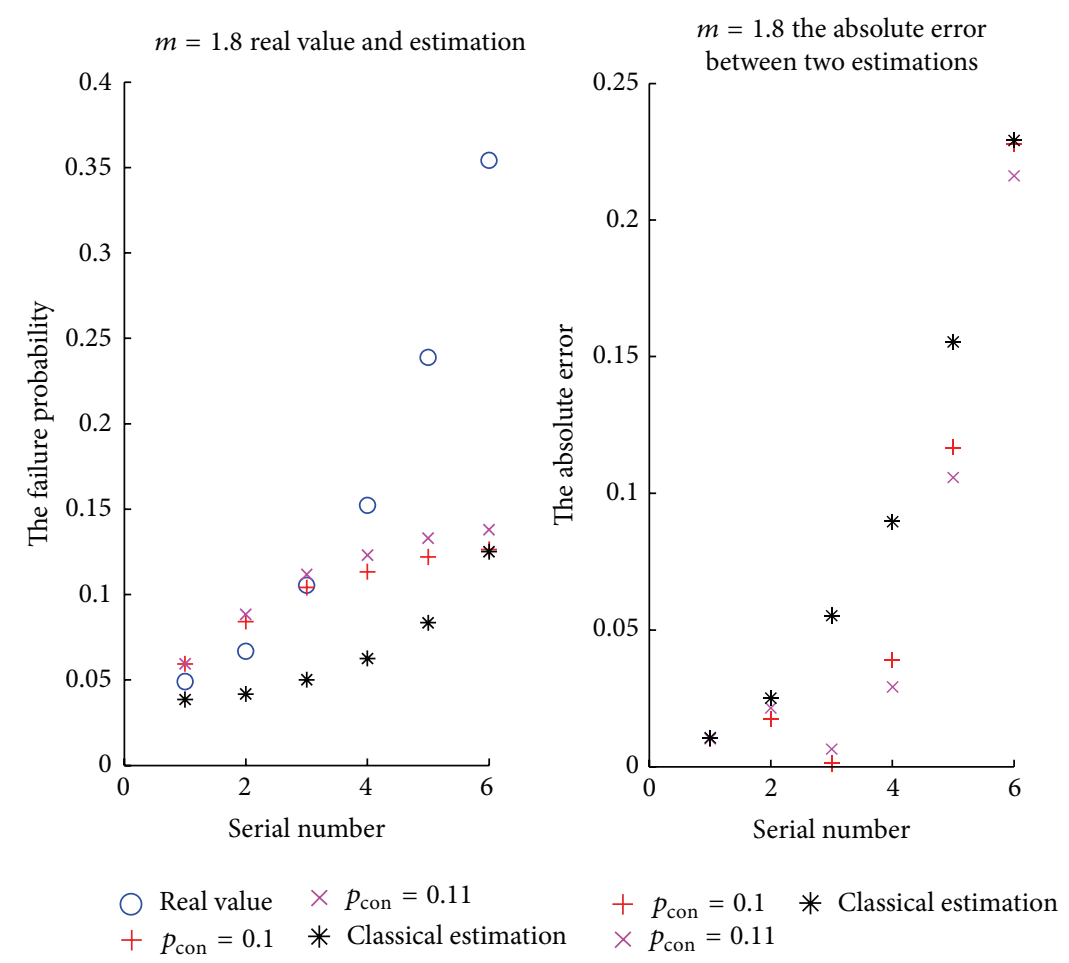

FIGURE 3: Comparison of failure probability among true value, estimator, and classical estimation value when $m=1.8$. 

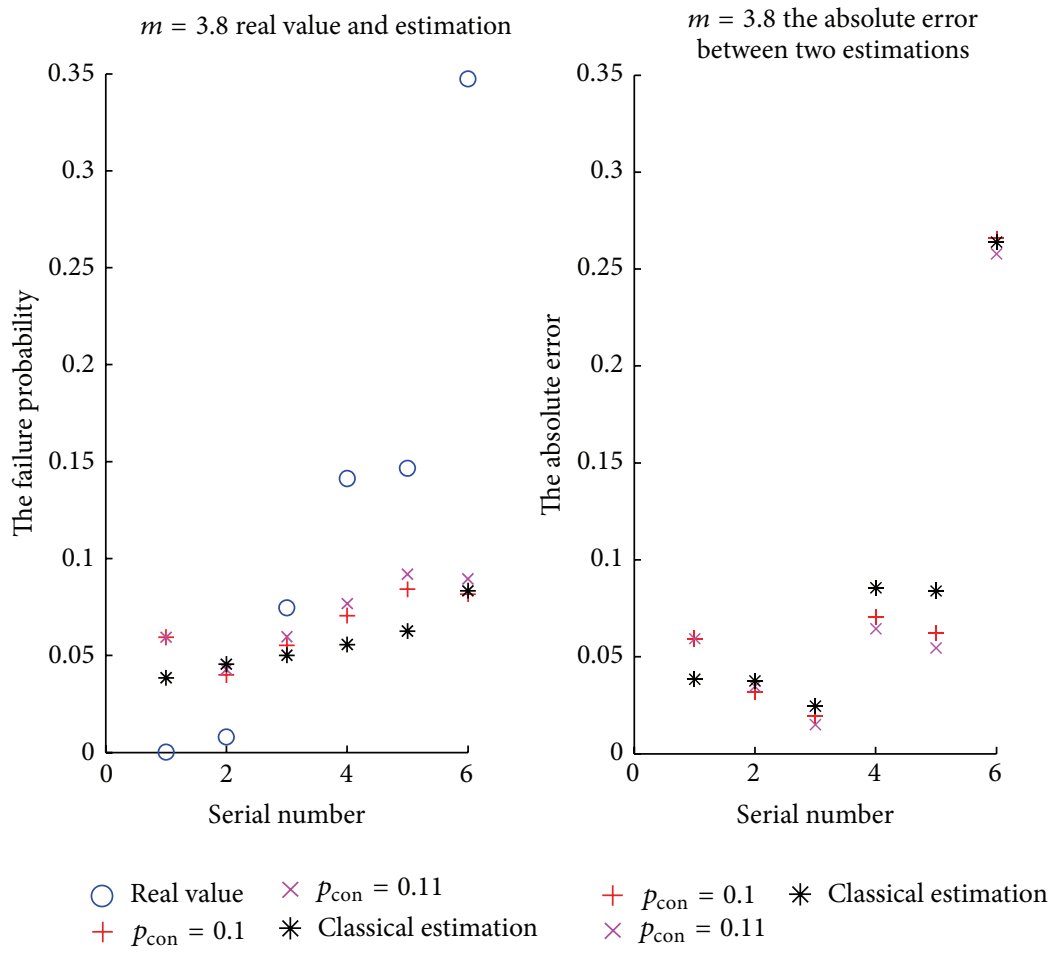

FIGURE 4: Comparison of failure probability among real value, estimator, and classical estimation value when $m=3.8$.

values of $p_{\text {upper }}$ are assigned to 0.3 and 0.25 to compare the effects of $p_{\text {upper. }}$. Figures 3 and 4 are generated by the Weibull distributions with the parameters $m=1.8, \eta=2800$ and $m=3.8, \eta=2800$, respectively. In the calculation of Figures 3 and 4 , we let $p_{\text {upper }}=0.25$, where the values of $p_{\text {con }}$ are assigned to 0.1 and 0.11 , to compare the effects of $p_{\text {con }}$. In all these figures, the left graph compares the estimates by our proposed method with those by the classical method with the true values; the right graph shows the absolute differences between the estimates by our proposed method and the true values, as well as the differences between the estimates by the classical method with the true values.

From the above comparisons, we find that

(1) $p_{\text {upper }}$ and $p_{\text {con }}$ have limited effects on the failure probability estimation, which validates the robustness of our proposed method;

(2) the estimations of our proposed method have less MSEs than those of the classical method, especially when the number of tests increases. The comparisons indicate that our method is more accurate than the classical method.

\section{Conclusion}

Reliability analysis based on zero-failure data attracts more and more attention as products become more reliable and very few failures are observed during testing. To solve the failure probability estimation problem in the Weibull distribution with zero-failure data, this paper presents a method of combining the decreasing function method with Bayesian theory to estimate the failure probability. The proposed method applies the concave and convex properties of the Weibull distribution with respect to the shape parameter, $m$, and provides the corresponding interval of failure probability, $p$. Then, the prior distribution of $p$ is constructed by using the decreasing function method, based on which the estimate of $p_{i}$ is calculated by applying the Bayesian method. A numerical example is presented to compare the estimations made by the proposed method and the classical method and the true values, which illustrates the validity and robustness of the proposed method.

\section{Notations}

CDF: Cumulative distribution function

PDF: Probability density function

MSE: Mean square error.

\section{Conflict of Interests}

The authors declare that there is no conflict of interests regarding the publication of this paper.

\section{Acknowledgments}

This research is supported by the projects of the National Natural Science Foundation of China (with Grant nos. 71371182 and 71401170) and the Research Project of National University of Defense Technology (with Grant no. JC13-0205). 


\section{References}

[1] E. L. Welker and M. Lipow, "Estimating the exponential failure rate from data with no failure events," in Proceedings of the Annual Reliability and Maintainability Symposium, pp. 420-427, 1974.

[2] H. F. Martz Jr. and R. A. Waller, "A bayes zero-failure(BAZE) reliability demonstration testing procedure," Journal of Quality Technology, vol. 11, no. 3, pp. 128-137, 1979.

[3] H. Qi, S. Ganesan, and M. Pecht, "No-fault-found and intermittent failures in electronic products," Microelectronics Reliability, vol. 48, no. 5, pp. 663-674, 2008.

[4] R. T. Bailey, "Estimation from zero-failure data," Risk Analysis, vol. 17, no. 3, pp. 375-380, 1997.

[5] W. Wang and D. R. Langanke, "Comparing two designs when the new design has few or no failures-is the new design better than previous one?" in Proceedings of the International Symposium on Product Quality and Integrity, pp. 322-325, January 2001.

[6] K. W. Miller, L. J. Morell, R. E. Noonan et al., "Estimating the probability of failure when testing reveals no failures," IEEE Transactions on Software Engineering, vol. 18, no. 1, pp. 33-43, 1992.

[7] P. Jiang, J.-H. Lim, M. J. Zuo, and B. Guo, "Reliability estimation in a Weibull lifetime distribution with zero-failure field data," Quality and Reliability Engineering International, vol. 26, no. 7, pp. 691-701, 2010.

[8] J. D. Chen, W. L. Sun, and B. X. Li, "The confidence limits in the case of no failure data," Acta Mathematicae Applicatae Sinica, vol. 18, no. 1, pp. 90-100, 1995 (Chinese).

[9] L. Wang and B. Wang, "Statistical analysis for no-failure datamodified likelihood function method," Mathematical Statistics and Applied Probability, vol. 11, no. 1, pp. 64-70, 1996 (Chinese).

[10] J. Ning, Reliability assessment on zero-failure data of liquid rocket engine [M.S. thesis], National University of Defense Technology, 2005 (Chinese).

[11] F. Li, Statistical analysis on zero-failure data [M.S. thesis], Anhui Normal University, 2006.

[12] S. Mao and C. Luo, "Reliability analysis of no failure data," Mathematic Statistics and Applied Probability, vol. 4, no. 4, pp. 489-506, 1989 (Chinese).

[13] D. Ling, Research on Weibull distribution and its applications in mechanical reliability engineering [Dissertation thesis], University of Electronic Science and Technology of China, 2010, (Chinese).

[14] M. Han and Y.-Q. Li, "Hierarchical Bayesian estimation of the products reliability based on zero-failure data," Journal of Systems Science and Systems Engineering, vol. 8, no. 4, pp. 467471, 1999.

[15] Z. Zhang, "Statistical analysis of data only one failure under normal case," Chinese Journal of Applied Probability, vol. 14, no. 2, pp. 185-190, 1998 (Chinese).

[16] M. Han, "Confidence limits of reliability parameters for weibull distribution," Journal of Mechanical Strength, vol. 31, no. 1, pp. 59-62, 2009 (Chinese).

[17] P. Jiang, X. Jia, and B. Guo, "Failure probability estimation in the Weibull distribution with zero-failure data," in Proceedings of the 8th IMA International Conference on Modelling in Industrial Maintenance and Reliability (MIMAR '14), pp. 131-136, 2014.

[18] L. Xiong, Statistical analysis of reliability about zero-failure data [M.S. thesis], Jiling University, 2004, (Chinese).
[19] D. C. Li and L. S. Lin, "A new approach to assess product lifetime performance for small data sets," European Journal of Operational Research, vol. 230, no. 2, pp. 290-298, 2013. 


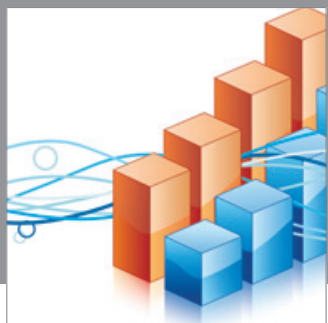

Advances in

Operations Research

mansans

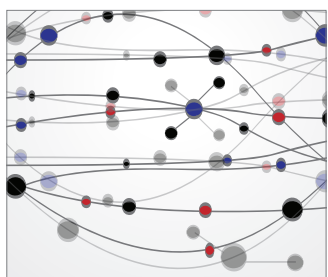

The Scientific World Journal
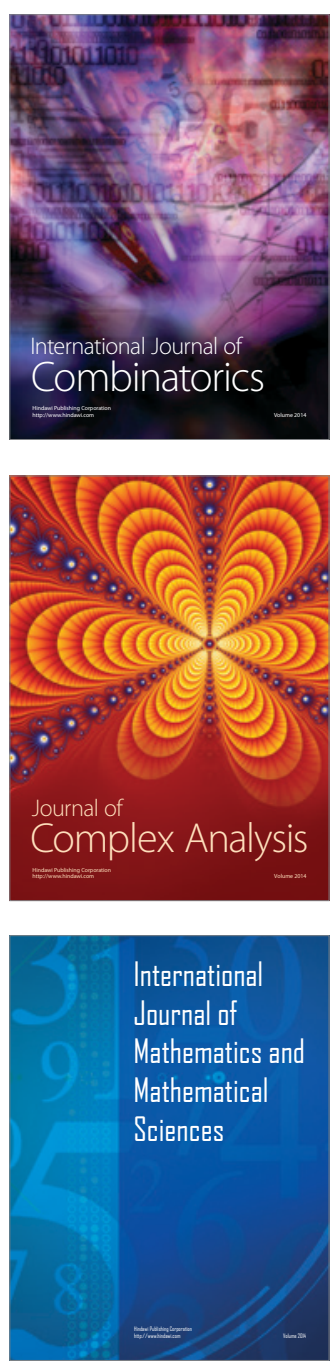
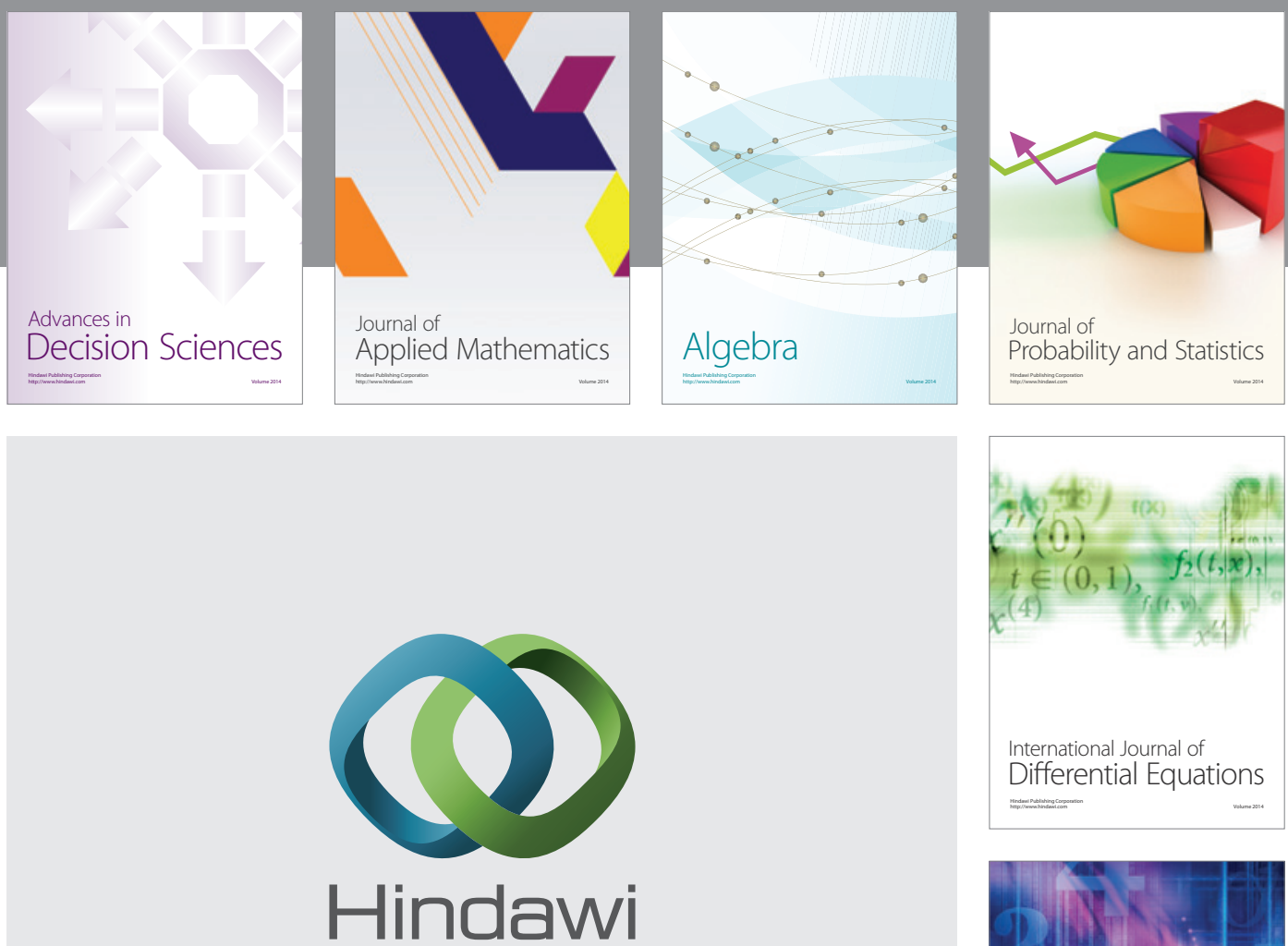

Submit your manuscripts at http://www.hindawi.com
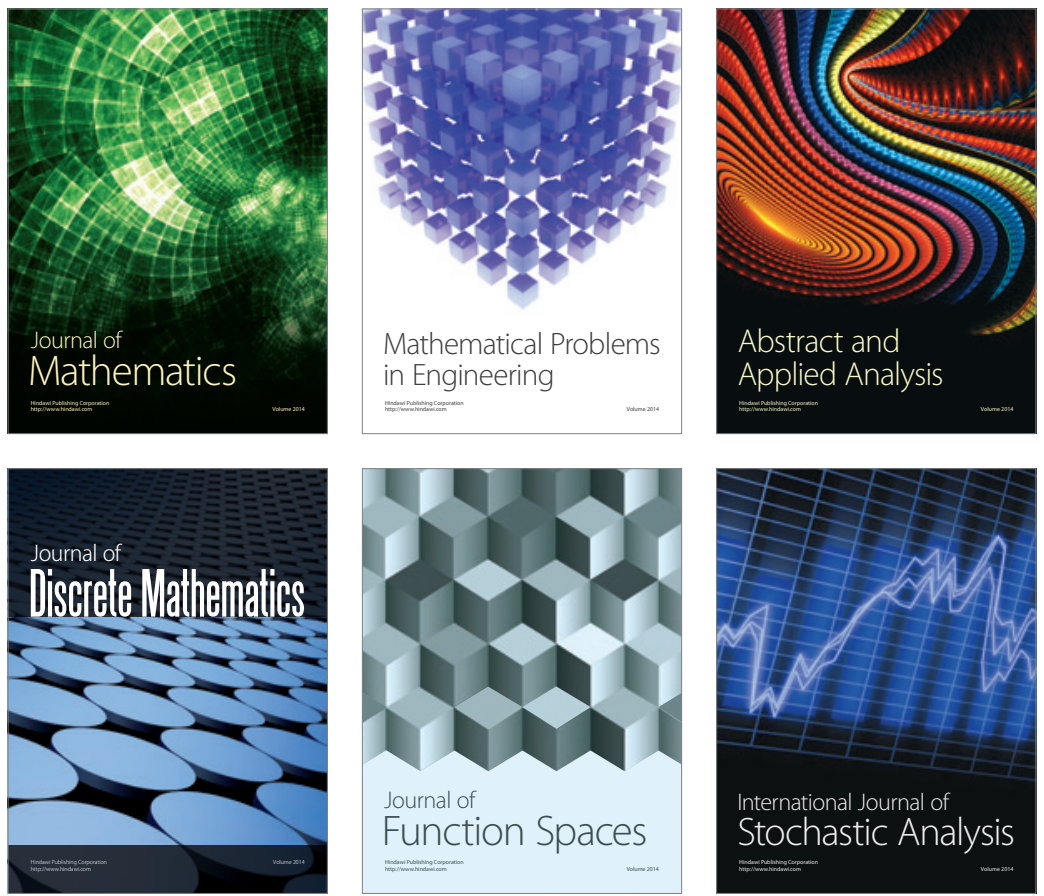

Journal of

Function Spaces

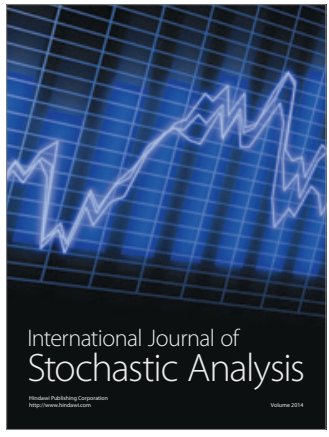

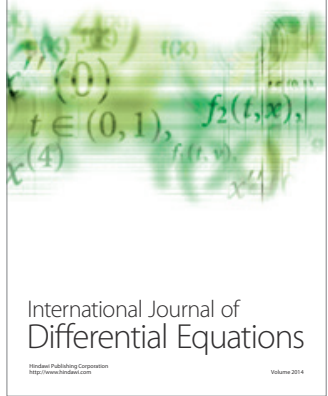
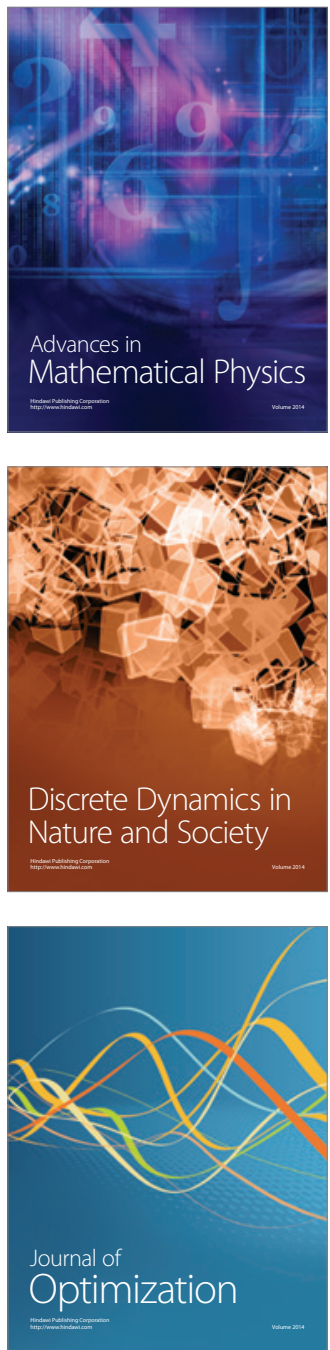